\title{
Beating of Friedel oscillations induced by spin-orbit interaction
}

\author{
S. M. Badalyan, ${ }^{1,2, *}$ A. Matos-Abiague, ${ }^{1}$ G. Vignale, ${ }^{3}$ and J. Fabian ${ }^{1}$ \\ ${ }^{1}$ Department of Physics, University of Regensburg, 93040 Regensburg, Germany \\ ${ }^{2}$ Department of Radiophysics, Yerevan State University, 1 A. Manoukian Street, Yerevan 375025, Armenia \\ ${ }^{3}$ Department of Physics and Astronomy, University of Missouri, Columbia, Missouri 65211, USA
}

(Received 25 April 2010; published 18 May 2010)

\begin{abstract}
By exploiting our recently derived exact formula for the Lindhard polarization function in the presence of Bychkov-Rashba (BR) and Dresselhaus (D) spin-orbit interaction (SOI), we show that the interplay of different SOI mechanisms induces highly anisotropic modifications of the static dielectric function. We find that under certain circumstances the polarization function exhibits doubly singular behavior. It leads to an intriguing phenomenon, beating of Friedel oscillations, which can be controlled by external fields. This effect is a general feature of systems with BR+D SOI and should be observed in structures with a sufficiently strong SOI.
\end{abstract}

DOI: 10.1103/PhysRevB.81.205314

PACS number(s): 71.70.Ej, 72.10.-d, 72.20.-i, 72.25.-b

Spin-orbit interaction (SOI) is of great interest for spintronic applications. ${ }^{1,2}$ Electron spin is not conserved in the presence of SOI, which allows for purely electric manipulation of spins. ${ }^{3,4}$ In conjunction with other carrier scattering mechanisms, SOI leads to intriguing phenomena. One of the major findings is the detection of the spin Hall effect, ${ }^{5-8}$ predicted long ago as an outcome of the interplay between SOI and electron-impurity scattering. ${ }^{9}$ In turn, electronelectron scattering mediates mutual transformations of spin and charge currents, occurring due to spin Coulomb drag in individual layers ${ }^{10,11}$ and due to spin Hall drag ${ }^{12}$ in electronic bilayers.

In zinc-blende semiconductor nanostructures the interplay between different mechanisms of SOI can itself have crucial consequences. In the presence of both Bychkov-Rashba ${ }^{13}$ (BR) and Dresselhaus ${ }^{14}$ (D) SOI the system possesses $C_{2 v}$ symmetry. The BR coupling strength $\alpha$ depends largely on the asymmetry of structure while the $\mathrm{D}$ coupling $\beta$ varies mainly with the thickness of structure. In the special case when the BR and D SOI strengths are adjusted ${ }^{15,16}$ to be equal, even higher $\mathrm{SU}(2)$ symmetry occurs in the system ${ }^{17}$ and various relaxation ${ }^{18}$ and optical ${ }^{19}$ properties of the system turned out to be identical to those in the absence of SOI. A remarkable demonstration of such suppression of SOI is the fresh experimental realization ${ }^{20}$ of the persistent spin helix.

Another distinct manifestation of the interplay of BR and D mechanisms is the SOI-induced anisotropy of the singleparticle spectrum, which modifies spin relaxation and transport properties of the system. ${ }^{21-23}$ Recently we have studied the influence of that anisotropy on the many-body response of a two-dimensional electron system (2DES). ${ }^{24}$ Our calculations have revealed a fine structure of the plasmon spectrum, which produces a striking asymmetric doublet of the structure factor versus momentum orientation. The joint action of BR and D SOI leads to dependence of the interchirality particle-hole continuum on direction. Thus, the plasmon propagation may be free in one direction, but strongly damped in a different direction, where the plasmon dispersion enters the particle-hole continuum. This creates a possibility of directional plasmon filtering, potentially useful for both spintronic and plasmonic devices.

In this paper we study the many-body response of $2 \mathrm{DES}$ in the presence of BR and D SOI in the static limit. By exploiting our recently derived exact formula for the Lindhard polarization function (PF), we reveal a highly anisotropic behavior of the static dielectric function. Particularly, the interchirality transitions strongly modify the singular behavior of the dielectric function. The sharp anisotropic Fermi surface makes the position of singularities dependent on momentum orientation and on the ratio of the BR and D SOI strengths, in addition to the usual dependence on the magnitude of momentum $q$. We find that PF shows a sharp cusp at $q<2 k_{F}\left(k_{F}\right.$ is the Fermi wave vector) for the momentum orientation along the $[1 \overline{1} 0]$ direction while in the perpendicular [110] direction, the singularity occurs at $q>2 k_{F}$. Most importantly, we observe that PF exhibits a doubly singular behavior - the singularities occur both at $q<2 k_{F}$ and $q>2 k_{F}$. As a direct consequence of this, we find that the Friedel oscillations propagate with two slightly different spatial frequencies and a beating phenomenon of Friedel oscillations takes place. The unique feature of our proposal is that it would allow us to continuously tune, through external fields, the spatial frequency of the oscillations. For example, at strictly equal SOI strengths, $\alpha=\beta$, only the second singularity survives. The Friedel oscillations become isotropic and the effect of SOI reduces to a simple renormalization of the singularity position.

The Hamiltonian of BR and D SOI in quantum wells of zinc-blende structure, grown on a (001) surface, is $H_{\mathrm{SO}}$ $=\alpha\left(\hat{\sigma}_{x} k_{y}-\hat{\sigma}_{y} k_{x}\right)+\beta\left(\hat{\sigma}_{x} k_{x}-\hat{\sigma}_{y} k_{y}\right)$, where $\hat{\sigma}_{x, y}$ are the Pauli matrices, $\vec{k}$ is the in-plane electron momentum with its magnitude $k$ and polar angle $\phi_{\mathbf{k}}$. The eigenvectors of the Hamiltonian $H=H_{0}+H_{\text {SOI }}$ with $H_{0}=\vec{k}^{2} / 2 m^{*}$ ( $m^{*}$ is the electron effective mass and $\hbar=1$ ) are

$$
\Psi_{\mu}(\vec{r})=\frac{1}{\sqrt{2 \mathcal{A}}}\left(\begin{array}{c}
i e^{-i \varphi} \\
\mu
\end{array}\right) e^{i \vec{k} \vec{r}}
$$

They correspond to the energy branches $E_{\mu}(\vec{k})=\frac{1}{2 m^{*}}$ $\left[\left[k+\mu \xi\left(\rho, \theta, \phi_{\mathbf{k}}\right)\right]^{2}-\xi\left(\rho, \theta, \phi_{\mathbf{k}}\right)^{2}\right]$, which are labeled by the chirality $\mu= \pm 1$. Here, $\mathcal{A}$ is the normalization area and the spinor phase is given by $\varphi\left(\alpha, \beta, \phi_{\mathbf{k}}\right)=\operatorname{Arg}\left[\alpha e^{i \phi_{\mathbf{k}}}+i \beta e^{-i \phi_{\mathbf{k}}}\right]$. The angle-dependent BR-D momentum is $\xi\left(\rho, \theta, \phi_{\mathbf{k}}\right)$ 

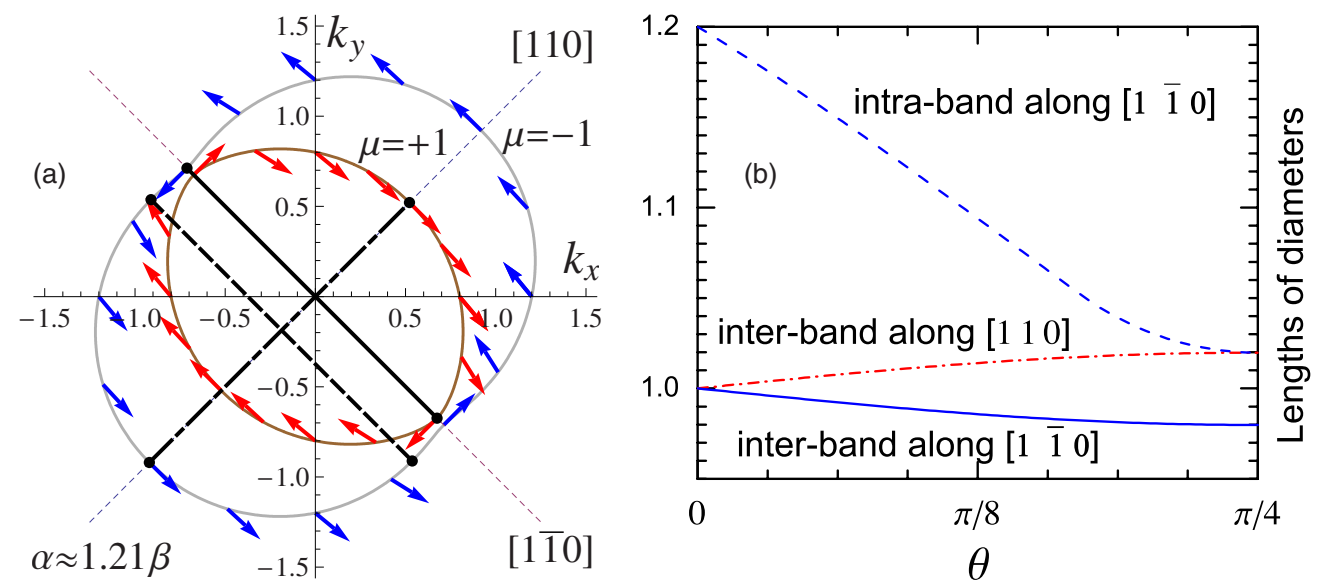

FIG. 1. (Color online) (a) The Fermi contour in the presence of BR+D SOI. Arrows indicate directions of spin. The thick solid and dashed lines are diameters along the [1 $1 \overline{1} 0]$ direction, connecting maximally distant points on the different chirality subbands and on the outer subband, respectively. The thick dashed-dotted line is the intersubband diameter in [110] direction. The BR and D strengths are related as $\alpha \approx 1.21 \beta$ with $\rho=0.2 k_{F}$ and the axes are in units of $k_{F}$. (b) The lengths of respective diameters of the Fermi contour in units of $2 k_{F}$ as a function of the parameter $\theta$ for $\rho=0.2 k_{F}$.

$=\rho \sqrt{1+\sin (2 \theta) \sin \left(2 \phi_{\mathbf{k}}\right)}$, where $\rho=m^{*} \sqrt{\alpha^{2}+\beta^{2}}$. The angle parameter $\theta$ is defined as $\tan \theta=\beta / \alpha$ and describes the relative strength of the BR and D SOI. The Fermi momenta of the chirality subbands are also angle dependent: $k_{F}^{\mu}\left(\rho, \theta, \phi_{\mathbf{k}}\right)$ $=\sqrt{2 m E_{F}+\xi\left(\rho, \theta, \phi_{\mathbf{k}}\right)^{2}}-\mu \xi\left(\rho, \theta, \phi_{\mathbf{k}}\right)$, where the total carrier density $n$ determines the Fermi energy $E_{F}=\left(\pi n-\rho^{2}\right) / m^{*}$. Figure 1 shows the anisotropic Fermi contour in the $\left(k_{x}, k_{y}\right)$ plane.

In the static limit the dielectric function $\varepsilon(\vec{q})=1$ $-v(q) \Pi(\vec{q})$, where $v(q)=2 \pi e^{2} /\left(\kappa_{0} q\right) F(q d)$ is the bare Coulomb interaction with $\kappa_{0}$ as the low-frequency dielectric constant. The form factor $F(q d)$ takes into account the transverse width $d$ of the quantum well. It goes as $1-\left(1 / 3-5 / 4 \pi^{2}\right) q d$ in the long-wavelength limit $q d \rightarrow 0$, and as $3 /\left(4 \pi^{2} q d\right)$, in the opposite limit $q d \rightarrow \infty$. The exact $\operatorname{PF} \Pi(\vec{q})$ can be expressed in terms of the noninteracting Lindhard response function $\Pi^{0}(\vec{q})$ as $\Pi(\vec{q})=\Pi^{0}(\vec{q})\left[1-v(q)\left(1-G_{+}(q)\right) \Pi^{0}(\vec{q})\right]^{-1}$. Here, the "charge-channel" local field factor $G_{+}(q)$ (Ref. 25) takes into account all electron correlations, related to the vertex corrections beyond the random-phase approximation. We neglect the effect of SOI on $G_{+}$.

The static Lindhard PF in the presence of SOI is defined as a sum over the indices $\mu$ and $\nu, \Pi^{0}(\vec{q})=\Sigma_{\mu, \nu= \pm 1} \Pi_{\mu \nu}^{0}(\vec{q})$, where

$$
\Pi_{\mu \nu}^{0}(\vec{q})=\int \frac{d \vec{k}}{(2 \pi)^{2}} \frac{f_{F}\left(E_{\mu}(\vec{k})\right)-f_{F}\left(E_{\mu \cdot \nu}(\vec{k}+\vec{q})\right)}{E_{\mu}(\vec{k})-E_{\mu \cdot \nu}(\vec{k}+\vec{q})} \mathcal{F}_{\nu}(\vec{k}, \vec{k}+\vec{q}) .
$$

Here, $\nu= \pm 1$ refers to the intrasubband and intersubband contributions to $\Pi^{0}(\vec{q})$ and $f_{F}\left(E_{\mu}(\vec{k})\right)$ are the Fermi distribution functions. The form factor $\mathcal{F}_{\nu}(\vec{k}, \vec{k}+\vec{q})$ comes from the product of bare vertices and describes the overlapping of spinor wave functions. It is given by $\mathcal{F}_{\nu}(\vec{k}, \vec{k}+\vec{q})=\frac{1}{2}$ $\left[1+\nu \cos \left(\Delta \varphi_{\mathbf{q}}\right)\right]$, where we define $\Delta \varphi_{\mathbf{q}}=\varphi\left(\alpha, \beta, \phi_{\mathbf{k}}\right)$ $-\varphi\left(\alpha, \beta, \phi_{\mathbf{k}+\mathbf{q}}\right)$. Notice that in contrast to the case with pure $\mathrm{BR}$ or D SOI, here PF depends additionally on the momen- tum orientation $\phi_{\mathbf{q}}$. Recently in Ref. 24 we have derived an exact formula for the $\mathrm{PF}$ in the presence of $\mathrm{BR}+\mathrm{D} \mathrm{SOI}$, which is omitted here for the sake of brevity. The results, obtained from this formula, are in agreement with the previous classic result by $\operatorname{Stern}^{26}$ and findings by Pletyukhov and Gritsev, ${ }^{27}$ respectively, in the limits of vanishing SOI and of pure BR SOI.

Further by exploiting this general formula for the $\mathrm{PF}^{24}$ we calculate the static PF and analyze the modifications induced by the $\mathrm{BR}+\mathrm{D}$ SOI. We use the realistic materials parameters for InAs quantum wells by taking $m^{*}=0.023 m_{0}, \kappa_{0}=14.55$, and the transverse width of the quantum well $d=15 \mathrm{~nm}$. We have also defined and will use in the following the dimensionless parameters $x=q / 2 k_{F}$ and $r=\rho / k_{F}$ with $k_{F}$ $=\sqrt{2 m^{*} E_{F}+\rho^{2}}$. In Fig. 2 we plot $\Pi^{0}(\vec{q})$ in units of the density of states at the Fermi level $g=m^{*} / 2 \pi$ as a function of $q$ for two different orientations of the momentum, $\phi_{\mathbf{q}}=\pi / 4$ and $\phi_{\mathbf{q}}=3 \pi / 4$, and for several values of the angle parameter $\theta$. The solid line represents PF in the absence of SOI, $r=0$, while the dashed line corresponds to $\operatorname{PF} \Pi_{B R D}^{0}(|\vec{q}|)$ in the case of the pure BR or D SOI, $\theta=0$ or $\pi / 2$, respectively. All the curves of $\Pi^{0}(\vec{q})$ show singular behavior at one or two values of $q$, determined by the lengths of diameters in Figs. 1(a) and 1(b). Independent of $\theta$, the curves, which refer to $\phi_{\mathbf{q}}$ $=3 \pi / 4$, exhibit a singularity at the wave vectors $q_{1 c}$ $=2 k_{F} \sqrt{1-r^{2} \sin 2 \theta}<2 k_{F}$ (cf. the solid line diameter in Fig. 1) with a maximum polarizability at the singular point, $\Pi_{1}^{\max }$, exceeding the maximum value of $\Pi_{B R D}^{0}(|\vec{q}|)$. At wave vectors $q_{2 c}>2 k_{F}$ PF develops a second point of nonanalyticity with a local maximum $\Pi_{2}^{\max }<2$. Here, $q_{2 c}=\max _{\phi_{\mathbf{k}}} Q\left(\phi_{\mathbf{k}}\right)$ with the function

$$
Q\left(\phi_{\mathbf{k}}\right)=\left|\left[\sin \left(\phi_{\mathbf{k}}\right)-\cos \left(\phi_{\mathbf{k}}\right)\right]\left[\bar{\xi}_{\mathbf{k}}+\sqrt{1+r^{2} \sin \left(2 \phi_{\mathbf{k}}\right) \sin (2 \theta)}\right]\right|
$$

(cf. the dashed line diameter in Fig. 1). Here, $\bar{\xi}_{\mathrm{k}}$ $=\xi\left(\rho, \theta, \phi_{\mathbf{k}}\right) / k_{F}$. For values of $\theta$ not far from $\pi / 4$, the singularity at $q_{2 c}$ becomes well pronounced while the singularity at $q_{1 c}$ turns into a sharp cusp. The curves in Fig. 2, which 

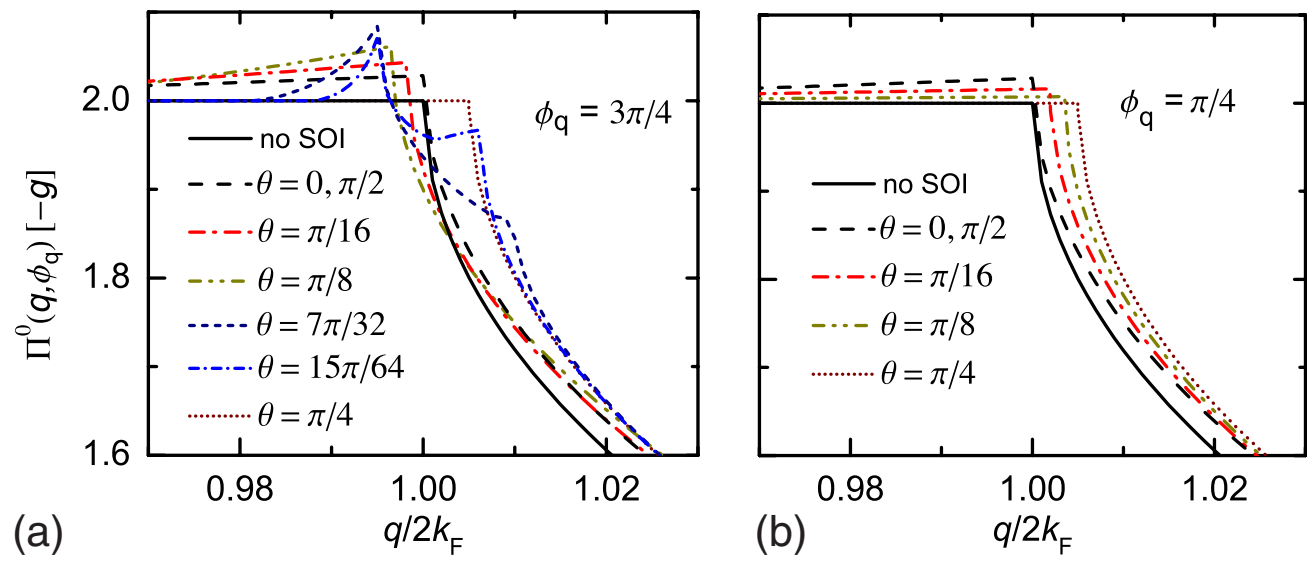

FIG. 2. (Color online) The static polarization function $\Pi^{0}(\vec{q})$ as a function of the momentum $q$ for its two orientations: (a) $\phi_{\mathbf{q}}=3 \pi / 4$ and (b) $\pi / 4$. The different curves correspond to different values of $\theta$, shown on the graph legends for $r=0.1$.

refer to $\phi_{q}=\pi / 4$, exhibit only one singularity at the position $q_{3 c}=2 k_{F} \sqrt{1+r^{2} \sin 2 \theta}>2 k_{F} \quad$ (cf. the dashed-dotted line diameter in Fig. 1) and the polarizability in the singular point varies within the $2<\Pi^{0}(\vec{q})<\Pi_{B R D}^{0}(|\vec{q}|)$ window. In all singular points the $\mathrm{PF}$ is continuous and its derivative is discontinuous. At the special values of $\theta=\pi / 4$ and $3 \pi / 4$ when $\alpha$ $= \pm \beta$, the form factor $\mathcal{F}_{\nu}$ ceases to depend on the wave vector $\vec{k}$ and reduces to the Kronecker symbol. In this case we find that the effect of SOI on the density response of a 2DES disappears for any value of $\phi_{\mathbf{q}}$. The only remaining modification reduces to a simple renormalization of the isotropic Fermi wave vector, $k_{F} \rightarrow k_{F c}=\sqrt{1+r^{2}} k_{F}$.

The electron-density deviation, generated by the perturbation of a single impurity, which is embedded at $R=0$ in the electron sheet in the $(x, y)$ plane, is determined by the static density response function via the relation

$$
\delta n(\vec{R})=\int \frac{d \vec{q}}{(2 \pi)^{2}} e^{i \vec{q} \vec{R}} V_{i}(q) \Pi(\vec{q}),
$$

where $V_{i}(q)$ is the Fourier transform of an isotropic impurity potential. Since in the presence of BR+D SOI PF is anisotropic, we can rewrite Eq. (2) as

$$
\frac{\delta n(\vec{R})}{n}=\frac{2}{\pi} \int_{0}^{\infty} d x x V_{i}(x) \int_{0}^{2 \pi} d \phi_{\mathbf{q}} e^{i \xi x \cos \left(\phi_{\mathbf{q}}-\phi_{\mathbf{R}}\right)} \Pi\left(x, \phi_{\mathbf{q}}\right),
$$

where $\xi=2 k_{F} R=\left(2 \sqrt{2} / r_{s}\right)\left(R / a_{B}\right)$ and $\phi_{\mathbf{R}}$ is the polar angle of the vector $\vec{R}, r_{s}=\sqrt{2} / k_{F} a_{B}$ is the dimensionless constant of electron-electron interaction, ${ }^{25}$ and $a_{B}$ is the effective Bohr radius. At large distances from the impurity, the integrand has a rapidly varying phase, $\xi x \cos \left(\phi_{\mathbf{q}}-\phi_{\mathbf{R}}\right)$, so the main contribution to the integral comes from the point where the phase is stationary and the exponential function $e^{i \xi x \cos \left(\phi_{\mathbf{q}-} \phi_{\mathbf{R}}\right)}$ oscillates less rapidly. The application of the method of stationary phase to the integral over $\phi_{\mathbf{q}}$ in Eq. (3) yields

$$
\frac{\delta n(\vec{R})}{n} \sim 4 \sqrt{\frac{2}{\pi \xi}} \int_{0}^{\infty} d x \sqrt{x} V_{i m p}(x) \Pi\left(x, \phi_{\mathbf{R}}\right) \cos \left(\frac{\pi}{4}-\xi x\right) .
$$

Here, we have used the relation $\Pi(x, 0)=\Pi(x, \pi)$, which is the case in the presence of BR+D SOI due to $C_{2 v}$ symmetry. Thus, at large distances from the impurity one can replace the second argument of PF $\phi_{\mathbf{q}}$ with $\phi_{\mathbf{R}}$.

In order to take the integration over $x$ we can exploit the Riemann-Lebesgue lemma, which says that if a function oscillates rapidly around zero then the integral of this function is small and the principal contribution to the integral is determined by the integrand behavior in the neighborhood of singular points. Therefore, one can reduce Eq. (4) to the following asymptotic expression for the density deviation:

$$
\frac{\delta n(\vec{R})}{n} \sim \frac{1}{g} \sum_{i} \sqrt{\frac{2 x_{i c}}{\pi \xi}} A\left(x_{i c}\right) \int d x \delta \Pi^{0}\left(x, x_{i c}\right) \cos \left(\frac{\pi}{4}-\xi x\right),
$$

where

$$
A\left(x_{i c}\right)=\frac{4 g V_{i m p}\left(x_{i c}\right)}{\left\{1-v\left(x_{i c}\right)\left[1-G_{+}\left(x_{i c}\right)\right] \Pi^{0}\left(x_{i c}, \phi_{\mathbf{R}}\right)\right\}^{2}},
$$

and $x_{i c}=x_{i c}\left(r, \theta, \phi_{\mathbf{q}}\right)$ denotes the position of the $i$ th singularity of PF. The increment of $\mathrm{PF} \delta \Pi^{0}\left(x, x_{i c}\right)=\Pi^{0}\left(x, \phi_{\mathbf{R}}\right)$ $-\Pi^{0}\left(x_{i c}, \phi_{\mathbf{R}}\right)$ near the singularity $x_{i c}$ can be represented as

$$
\delta \Pi^{0}\left(x, x_{i c}\right) \approx-g \vartheta\left[ \pm\left(x-x_{i c}\right)\right] \frac{a_{i}}{x}\left|x^{2}-x_{i c}^{2}\right|^{\alpha_{ \pm}, i},
$$

where $\vartheta(x)$ is the unit step function and the signs \pm correspond to the function below $\left(x<x_{i c}\right)$ and above $\left(x>x_{i c}\right)$ the singularity $x_{i c}$. The critical exponents $\alpha_{ \pm, i}$ and the coefficients $a_{i}$ describe the power-law behavior and the maximum polarizability at the singular points. In Eq. (5) we have assumed that the nonanalytical behavior of the interacting PF $\Pi(\vec{q})$ is completely determined by its noninteracting part $\Pi^{0}(\vec{q}) \cdot{ }^{25}$ Substituting Eq. (7) into Eq. (5) and making use the Lighthill theorem, ${ }^{28}$ after the integration over $x$ we get 

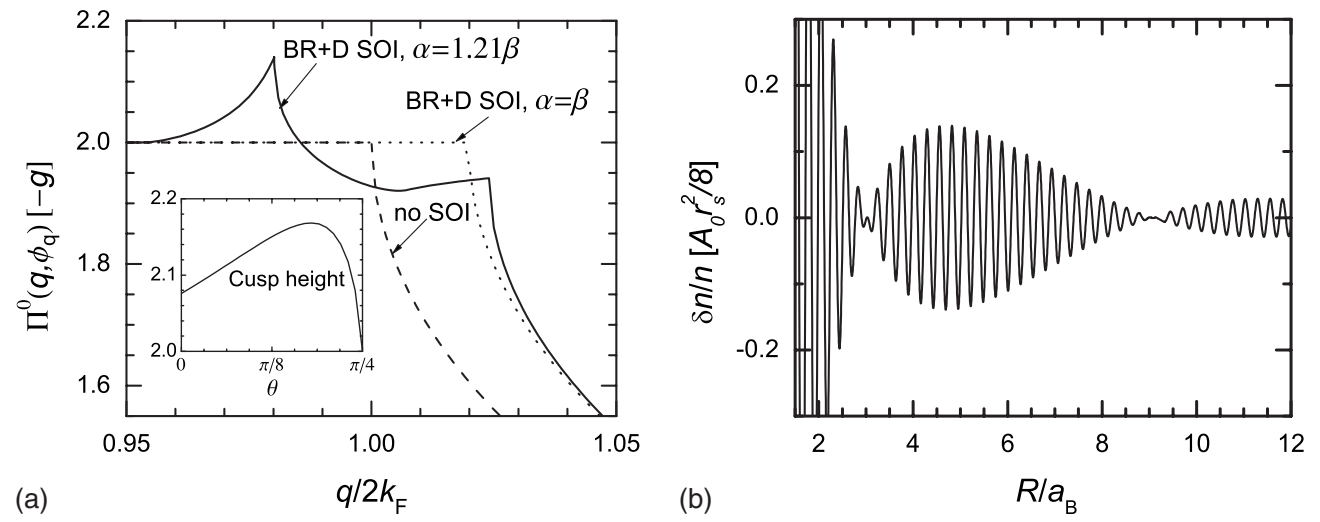

FIG. 3. (a) The doubly singular polarization function for $\alpha=1.21 \beta$ with $\rho=0.2 k_{F}$ (solid line). Inset shows the height of cusp vs $\theta$. The dotted and dashed curves represent $\Pi^{0}(\vec{q})$ for $\alpha=\beta$ with $\rho=0.2 k_{F}$ and $\rho=0$, respectively. (b) Beating of Friedel oscillations, induced by the doubly singular behavior of $\Pi^{0}(\vec{q})$ in (a). The electron density $n=2 \times 10^{16} \mathrm{~m}^{-2}$.

$$
\frac{\delta n(\vec{R})}{n} \sim-\frac{2 A_{0}}{\sqrt{\pi \xi}} \sum_{ \pm, i} \frac{\left(\alpha_{ \pm, i}\right) !}{\xi^{1+\alpha_{ \pm, i}}} \cos \left[\xi x_{i c}+\frac{\pi}{2}\left(\alpha_{ \pm, i}+\frac{1}{2}\right)\right] .
$$

Here, we take into account that the smooth functions $A\left(x_{i c}\right)$ and $a_{i}$ do not depend on the large parameter $\xi$, so that one can approximate $a_{i} A\left(x_{i c}\right) \approx 2 A(1) \equiv A_{0}$.

As we have already discussed, PF can qualitatively change its singular behavior, depending on the ratio of the BR and D SOI coupling strengths as well as on the momentum orientation. Accordingly, the Friedel oscillations, given by Eq. (8), can exhibit completely unique features. For instance, at $\theta=\pi / 8 \mathrm{PF}$ for two orthogonal orientations, $\phi_{\mathbf{q}}$ $=\pi / 4$ and $\phi_{\mathbf{q}}=3 \pi / 4$, shows singularities, respectively, at the positions $x_{+c}$ and $x_{-c}$ with $x_{ \pm c} \approx 1 \pm \delta_{c}$. It is clear that the phase difference between these two orientations is about $\left(x_{+c}-x_{-c}\right) \xi=\left(4 \sqrt{2} / r_{s}\right) \delta_{c}\left(R / a_{B}\right)$ and can result in a striking difference in the behavior of Friedel oscillations at the distance $R / a_{B}$ on the order of $r_{s} / 4 \sqrt{2} \delta_{c}$. In InAs samples with the electron density $n=10^{16} \mathrm{~m}^{-2}$, we have $r_{s} \approx 0.12$ and for $r=0.1$ taking $\delta_{c}=0.5 \times 10^{-2}$ we obtain that the Friedel oscillations in the $\phi_{\mathbf{R}}=\pi / 4$ and $\phi_{\mathbf{R}}=3 \pi / 4$ directions are in antiphase at the distance on the order of $R \leqq 5 a_{B}$.

Another interesting effect appears when the PF exhibits the doubly singular behavior (cf. the curves with $\theta=7 \pi / 32$ or $7 \pi / 32$ in Fig. 2). In Fig. 3(a) we illustrate the doubly singular behavior of PF separately for $r=0.2$. The inset shows the height of the cusp at $q=q_{1 c}$ [cf. the solid curve in Fig. 1(b)] versus $\theta$. As seen, $\Pi_{1}^{\max }=\Pi_{B R D}^{0}$ at $\theta=0$ and increases with $\theta$ up to its maximum $\Pi_{1}^{\max } \approx 2.17$ at about $\theta$ $=0.18 \pi$. With a further increase in $\theta, \Pi_{1}^{\max }$ drops sharply to its value in the absence of SOI, $\Pi_{1}^{\max }=2$, at $\theta=\pi / 4$. By fitting numerically the singularities of the exact $\mathrm{PF}$, we have established with numerical certainty that the critical exponents in the power-law behavior in Eq. (7) are (cf. Ref. 27) $\alpha_{-, 1,2}=1$ and $\alpha_{+, 1,2}=1 / 2$, respectively, for the left and right sides of both singularities at $x_{1 c}=1-\delta_{1 c}$ and $x_{2 c}=1+\delta_{2 c}$. Hence, in the limit of large $\xi$ the contributions to $\delta n(\vec{R})$, coming from the left-side neighborhood of both singularities, are small. Thus, the density deviation at large distances from the impurity can be reduced to the following simple form:

$$
\frac{\delta n(\vec{R})}{n} \sim \frac{A_{0}}{\xi^{2}}\left[\sin \left(1-\delta_{1 c}\right) \xi+\sin \left(1+\delta_{1 c}\right) \xi\right] .
$$

It is clear that due to the existence of two singularities at $x_{1 c}=1-\delta_{1 c}$ and $x_{2 c}=1+\delta_{2 c}$, the Friedel oscillations $\sin \left(1-\delta_{1 c}\right) \xi$ and $\sin \left(1+\delta_{1 c}\right) \xi$ propagate with two quite close spatial frequencies and a beating phenomenon of Friedel oscillations can be observed at the beat frequency $\sqrt{2}$ $\left(\delta_{1 c}+\delta_{2 c}\right) / r_{s}$. Figure 3(b) illustrates the first two destructive interferences of the Friedel oscillations that occur at $R$ $\sim 3 a_{B}$ and $9 a_{B}$. Taking into account higher-order terms in $\xi$ and $\rho$ will partially smooth the interference picture; however, the beating of Friedel oscillations as a distinct modulation of the density deviation is a stable feature of systems with BR $+\mathrm{D}$ SOI and should be observable in experiment. Notice also that in samples with a stronger SOI such as HgTe quantum wells, the separation $\delta_{1 c}+\delta_{2 c}$ between the singularities increases, which will essentially facilitate the experimental detection of the destructive interferences.

In conclusion, we have calculated the static response of a 2DES in the presence of joint BR and D SOI. We find that one of the main modifications is the induced shift of the singularity position of the static PF, which is in opposite directions for orthogonal momentum orientations. This results in a strong anisotropy of the Friedel oscillations. More interestingly, we have shown that in certain situations the PF exhibits a doubly singular behavior, which generates a unique phenomenon - the beating of Friedel oscillations. This intriguing prediction exemplifies how usually weak SOI can generate a qualitatively new and physically robust effect as a measurable signature of the many-body response of a 2DES. Because this effect arises at the interface of a semiconductor, we believe that it should be directly observable through tunneling microscopy imaging ${ }^{29,30}$ of the density distribution around an impurity.

We acknowledge support from EU Grant No. PIIF-GA2009-235394 (S.M.B.), SFB Grant No. 689, and NSF Grant No. DMR-0705460 (G.V.). 
*samvel.badalyan@physik.uni-regensburg.de

${ }^{1}$ J. Fabian, A. Matos-Abiague, C. Ertler, P. Stano, and I. Žutič, Acta Phys. Slov. 57, 565 (2007).

${ }^{2}$ I. Žutić, J. Fabian, and S. Das Sarma, Rev. Mod. Phys. 76, 323 (2004).

${ }^{3}$ D. Grundler, Phys. Rev. Lett. 84, 6074 (2000).

${ }^{4}$ J. Nitta, T. Akazaki, H. Takayanagi, and T. Enoki, Phys. Rev. Lett. 78, 1335 (1997).

${ }^{5}$ S. Murakami, N. Nagosa, and S.-C. Zhang, Science 301, 1348 (2003).

${ }^{6}$ Y. K. Kato, R. C. Myers, A. C. Gossard, and D. D. Awschalom, Science 306, 1910 (2004).

${ }^{7}$ J. Wunderlich, B. Kaestner, J. Sinova, and T. Jungwirth, Phys. Rev. Lett. 94, 047204 (2005).

${ }^{8}$ V. Sih, R. C. Myers, Y. K. Kato, W. H. Lau, A. C. Gossard, and D. D. Awschalom, Nat. Phys. 1, 31 (2005).

${ }^{9}$ M. I. D'yakonov and V. I. Perel', JETP Lett. 13, 144 (1971).

${ }^{10}$ C. P. Weber, N. Gedik, J. E. Moore, J. Orenstein, J. Stephens, and D. D. Awschalom, Nature (London) 437, 1330 (2005).

${ }^{11}$ S. M. Badalyan, C. S. Kim, and G. Vignale, Phys. Rev. Lett. 100, 016603 (2008).

${ }^{12}$ S. M. Badalyan and G. Vignale, Phys. Rev. Lett. 103, 196601 (2009).

${ }^{13}$ Yu. Bychkov and E. I. Rashba, JETP Lett. 39, 78 (1984).

${ }^{14}$ G. Dresselhaus, Phys. Rev. 100, 580 (1955).

${ }^{15}$ S. D. Ganichev, V. V. Bel'kov, L. E. Golub, E. L. Ivchenko, Petra Schneider, S. Giglberger, J. Eroms, J. De Boeck, G. Borghs, W. Wegscheider, D. Weiss, and W. Prettl, Phys. Rev. Lett. 92, 256601 (2004)

${ }^{16}$ S. Giglberger, L. E. Golub, V. V. Bel'kov, S. N. Danilov, D.
Schuh, C. Gerl, F. Rohlfing, J. Stahl, W. Wegscheider, D. Weiss, W. Prettl, and S. D. Ganichev, Phys. Rev. B 75, 035327 (2007).

${ }^{17}$ B. A. Bernevig, J. Orenstein, and S. C. Zhang, Phys. Rev. Lett. 97, 236601 (2006).

${ }^{18}$ J. Schliemann, J. C. Egues, and D. Loss, Phys. Rev. Lett. 90, 146801 (2003)

${ }^{19}$ J. A. Maytorena, C. López-Bastidas, and F. Mireles, Phys. Rev. 74, 235313 (2006).

${ }^{20}$ J. D. Koralek, C. Weber, J. Orenstein, B. A. Bernevig, S. C. Zhang, S. Mack, and D. D. Awschalom, Nature (London) 458, 610 (2009).

${ }^{21}$ N. S. Averkiev and L. E. Golub, Phys. Rev. B 60, 15582 (1999).

${ }^{22}$ P. Stano and J. Fabian, Phys. Rev. Lett. 96, 186602 (2006).

${ }^{23}$ J. Moser, A. Matos-Abiague, D. Schuh, W. Wegscheider, J. Fabian, and D. Weiss, Phys. Rev. Lett. 99, 056601 (2007).

${ }^{24}$ S. M. Badalyan, A. Matos-Abiague, G. Vignale, and J. Fabian, Phys. Rev. B 79, 205305 (2009).

${ }^{25}$ G. F. Giuliani and G. Vignale, Quantum Theory of the Electron Liquid (Cambridge University Press, Cambridge, England, 2005).

${ }^{26}$ F. Stern, Phys. Rev. Lett. 18, 546 (1967).

${ }^{27}$ M. Pletyukhov and V. Gritsev, Phys. Rev. B 74, 045307 (2006).

${ }^{28}$ M. J. Lighthill, Einführung in die Theorie der Fourier-Analysis und der Verallgemeinerten Funktionen (Hochschultaschenbü cher-Verlag, Mannheim, 1966).

${ }^{29}$ S. Becker, M. Liebmann, and Markus Morgenstern, DPG Spring Meeting 2010, Regensburg, Germany (unpublished), p. 332.

${ }^{30}$ K. Hashimoto, C. Sohrmann, J. Wiebe, T. Inaoka, F. Meier, Y. Hirayama, R. A. Romer, R. Wiesendanger, and M. Morgenstern, Phys. Rev. Lett. 101, 256802 (2008). 